\title{
Lattice study on two-color QCD with six flavors of dynamical quarks
}

\author{
M. Hayakawa ${ }^{* a}$, K.-I. Ishikawa ${ }^{b c}$, Y. Osaki ${ }^{b}$, S. Takeda ${ }^{d}$ and N. Yamada ${ }^{e, f}$ \\ ${ }^{a}$ Department of Physics, Nagoya University, Nagoya 464-8602, Japan \\ ${ }^{b}$ Department of Physics, Hiroshima University, Higashi-Hiroshima 739-8526, Japan \\ ${ }^{c}$ RIKEN Advanced Institute for Computational Science, Kobe, Hyogo 650-0047, Japan \\ ${ }^{d}$ School of Mathematics and Physics, College of Science and Engineering, Kanazawa University, \\ Kakuma-machi, Kanazawa, Ishikawa 920-1192, Japan \\ ${ }^{e}$ High Energy Accelerator Research Organization (KEK), Tsukuba, Ibaraki 305-0801, Japan \\ ${ }^{f}$ The Graduate University for Advanced Studies, Tsukuba, Ibaraki 305-0801, Japan
}

\begin{abstract}
We study the dynamics of SU(2) gauge theory with $N_{F}=6$ Dirac fermions by means of lattice simulation to investigate if they are appropriate to realization of electroweak symmetry breaking. The discrete analogue of beta function for the running coupling constant defined under the Schrödinger functional boundary condition are computed on the lattices up to linear size of $L / a=24$ and preclude the existence of infrared fixed point below $g^{2} \sim 7.6$. Gluonic observables such as heavy quark potential, string tension, Polyakov loop suggest that the target system is in the confining phase even in the massless quark limit.
\end{abstract}

The 30th International Symposium on Lattice Field Theory

July 24-29, 2012

Cairns, Australia

${ }^{*}$ Speaker. 
After the seminal work [1], great attention has been paid to the possibility to study nearly conformal dynamics of gauge theory by means of lattice simulation, which are expected to trigger electroweak symmetry breaking. So far, many works have been involved in the calculations on $\mathrm{SU}(3)_{\mathrm{C}}$ gauge theory with $N_{F}$ Dirac fermions in the fundamental representation. Recently, it was pointed out that three-color QCD with ten-flavors may be conformal in the infrared limit with large mass anomalous dimension [2,3]. However, large volume simulations indicated that chiral symmetry breaking occurs in the twelve-flavor system [4]. The large scale simulation via MonteCarlo renormalization group [5] will be effective to extract the genuine dynamics of the system.

Here we focus on a series of $\mathrm{SU}(2)_{\mathrm{C}}$ gauge theories with $N_{F}$ Dirac fermions in the fundamental representation, and try to find out a gauge system with the critical number of flavors $N_{F}^{\text {crt }}$, at which the chiral condensate $\bar{\psi} \psi$ gets large anomalous dimension. SU(2) has a symplectic form so that its fundamental representation is pseudo-real, and that the chiral symmetry of $N_{F}$-flavor system is enhanced to $\mathrm{SU}\left(2 N_{F}\right) \supset \mathrm{SU}\left(N_{F}\right)_{\mathrm{L}} \times \mathrm{SU}\left(N_{F}\right)_{\mathrm{R}} \times \mathrm{U}(1)_{\mathrm{V}}$. If chiral symmetry is spontaneously broken, the plausible unbroken symmetry subgroup is $\operatorname{SP}\left(2 N_{F}\right)$. But, this enhanced unbroken symmetry may contain the electroweak symmetry $G_{\mathrm{W}}=\mathrm{SU}(2)_{\mathrm{L}} \times \mathrm{U}(1)_{\mathrm{Y}}$ depending on the representation of new "quarks" under $G_{\mathrm{W}}$. It is thus inevitable to examine the vacuum alignment issue [6], i.e., whether $G_{\mathrm{W}}$ is broken or not. The transition between confinement and deconfinement is argued to be second-order in pure Yang-Mills theory with $\mathrm{SU}(2)_{\mathrm{C}}$ [7] while it is first-order for $\mathrm{SU}(3)_{\mathrm{C}}$. Therefore, $\mathrm{SU}(2)_{\mathrm{C}}$ chiral dynamics can differ even qualitatively from those of $\mathrm{SU}(3)_{\mathrm{C}}$, in particular at $N_{F}^{\text {crt }}$.

Actually, two-color QCD has also been studied thus far. Iwasaki et al. showed that $N_{F}=$ 3 system is conformal in the infrared (IR) limit [8] through study of phase structure of Wilson fermions, while the perturbatively calculated $\beta$ function suggests that $6 \leq N_{F}^{\text {crt }} \leq 8$. Afterwards, running gauge coupling constant has been calculated nonperturbatively for two-color QCD with six-flavors [9-11], and eight-flavors [12], implying that those systems are conformal in the IR limit $[9,11,12]$.

We note that $\mathrm{SU}(2)_{\mathrm{L}}$ gauge theory with three generations of quarks and leptons is exactly the system of our concern here, the two-color QCD with six massless Dirac fermions. Our question is if the quantum-mechanical dynamics of the presumed copy of the existing gauge symmetry and fermionic matters but with large $\Lambda$ " $\mathrm{QCD}^{\prime \prime}$ could play the role of triggering spontaneous breakdown of the electroweak symmetry, $\mathrm{SU}(2)_{\mathrm{L}} \times \mathrm{U}(1)_{\mathrm{Y}}$.

The purpose of this article is to report our first result for the dynamical features of twocolor QCD with six-flavors according to the simulation in the framework of lattice gauge theory. Throughout this work, the standard Wilson plaquette gauge action with unimproved Wilson fermions is used for simulation.

We measure $g_{\mathrm{SF}}\left(L / a, g_{0}^{2}\right)$ for sets of $\left(L / a, g_{0}^{2}=\frac{4}{\beta}\right)$ defined under the Schrödinger functional boundary condition with the twist angle for the quark fields set to 0 [13]. Compared to the preceding work [9], computation on larger lattices, $L / a=6,8,12,16,18$ and 24, is done with fine tuning of the critical value of hopping parameter for every pair of $\left(L / a, g_{0}^{2}\right)$. Data are fit to the presumed 
functional form

$$
\frac{g_{0}^{2}}{g_{\text {fit }}^{2}\left(L / a, g_{0}^{2}\right)}=\frac{1-a_{L / a}^{(1)} g_{0}^{4}}{1+p_{L / a, 1} g_{0}^{2}+\sum_{k=2}^{N} a_{L / a}^{(k)} g_{0}^{2 k}}
$$

where the coefficient $p_{L / a, 1}$ is obtained by the one-loop calculation. Using the fit result $g_{\text {fit }}^{2}\left(l, g_{0}^{2}\right)$, the discrete beta function [14]

$$
\left.B^{(s)}\left(u ; l_{1} \mapsto l_{2}=s l_{1}\right) \equiv \frac{1}{g_{\mathrm{fit}}^{2}\left(l_{2}=s l_{1}, g_{0}^{2}\right)}\right|_{u=g_{\mathrm{fit}}^{2}\left(l_{1}, g_{0}^{2}\right)}-\frac{1}{u},
$$

for a fixed step scaling factor $s$ can be calculated.

Figure 1 shows our result for its continuum limit $B(u, s=2)$ taken in the same manner as in Ref. [15]. The result precludes the existence of infrared fixed point $u_{\star}$ below 7.6. We are also trying to calculate the anomalous dimension of the chiral condensate $\bar{\psi} \psi$. We find that the result is roughly consistent with the perturbative prediction and the systematic uncertainties are under examination.

The above result suggests that $N_{F}=6$ is not far from $N_{F}^{\text {crt }}$ and motivates us to investigate the theory further from a different point of view. We thus study the dependence of mesonic spectrum on quark masses. First, in order to see the phase structure as a statistical

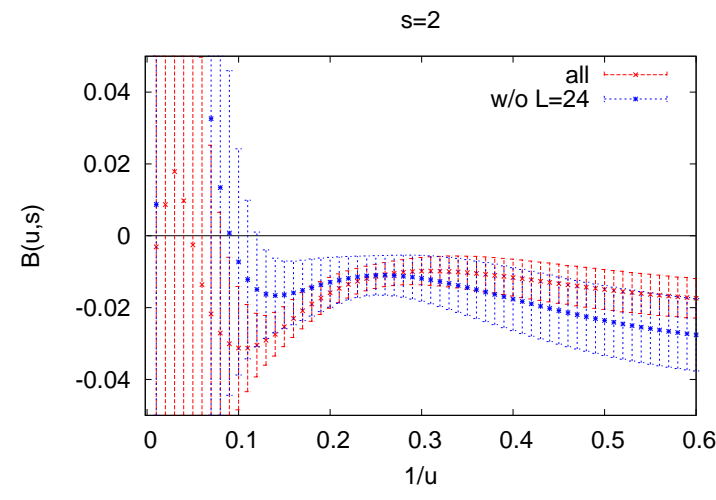

Figure 1: Discrete beta function for the step scaling $s=2$. The horizontal axis is the inverse of squared renormalized coupling constant. Red plots were obtained from all data, while blue plots were obtained from data without $L / a=24$.

system and fix the simulation parameters, we performed a scan on the $(\beta, \kappa)$-plane with relatively small lattices. Figure 2 shows the plaquette $\langle W\rangle$ as a function of $1 / \kappa$ on $8^{3} \times 24$ and $8^{3} \times 32$. For $\beta \lesssim 1.7$, there is a region of $\kappa$ in which $\langle W\rangle$ changes rapidly.

Thus, we choose $\beta=2.0$ to simulate larger lattices for our target system.

Figure 3 shows the masses of the lightest pseudoscalar and vector mesons, where the dependence on $\kappa$ is translated to that on $a_{\mathrm{PCAC}}$ via Figure 4 and the following properties are observed:

(1) The meson masses are bounded from below at small $\operatorname{am}_{\mathrm{PCAC}}$. The lower bound $a m_{\psi}^{\mathrm{sat}}(L / a)$ depends on $L / a$.

(2) Even for small $a m_{\mathrm{PCAC}}$, the ratio of the mass of the vector meson to that of the pseudoscalar meson is not far from 1. Figure 5 shows that the scalar meson is paired with the axial-vector meson ${ }^{1}$, and that their masses are well above those of the lightest pseudoscalar and vector mesons before saturation occurs.

\footnotetext{
${ }^{1}$ The fit is performed for the two-point correlation functions in the scalar and axial-vector channels before substantial fluctuation sets in at large $t / a$.
} 


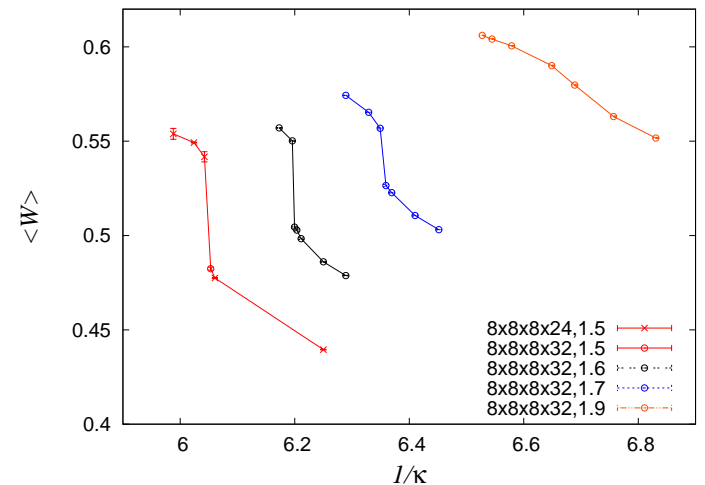

Figure 2: Plaquette, $\langle W\rangle$, versus $1 / \kappa$ on the lattices of spatial linear size $L / a=8$ with $\beta=1.5,1.6,1.7$ and 1.9 .

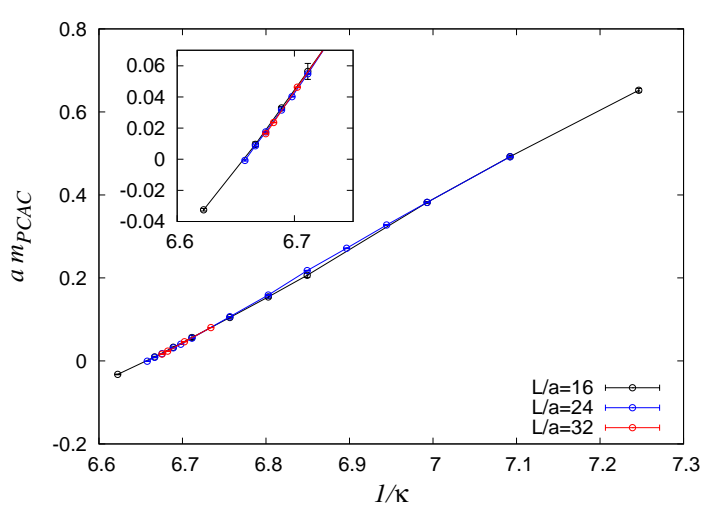

Figure 4: $\mathrm{PCAC}$ mass $m_{\mathrm{PCAC}}$ versus $1 / \kappa$ at $\beta=$ 2.0. The inset zooms in the vanishing $m_{\mathrm{PCAC}}$ region. Finite size effect is negligibly small.

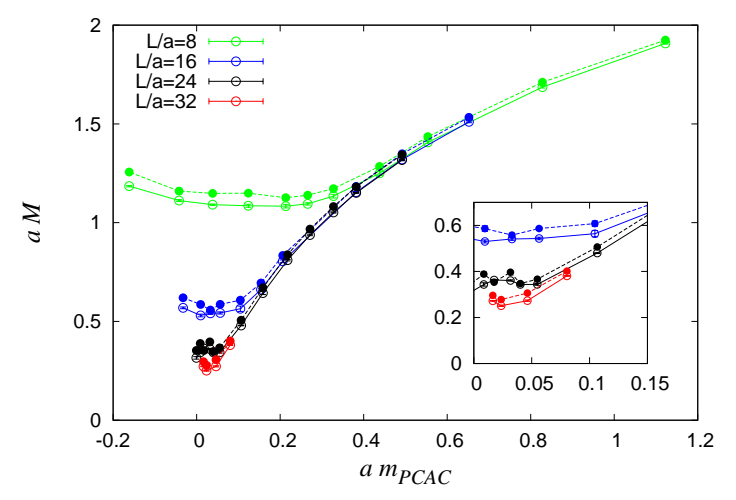

Figure 3: Lightest pseudoscalar (o) and vector $(\bullet)$ meson masses versus PCAC mass $m_{\mathrm{PCAC}}$ in lattice unit at $\beta=2.0$ on four different volumes.

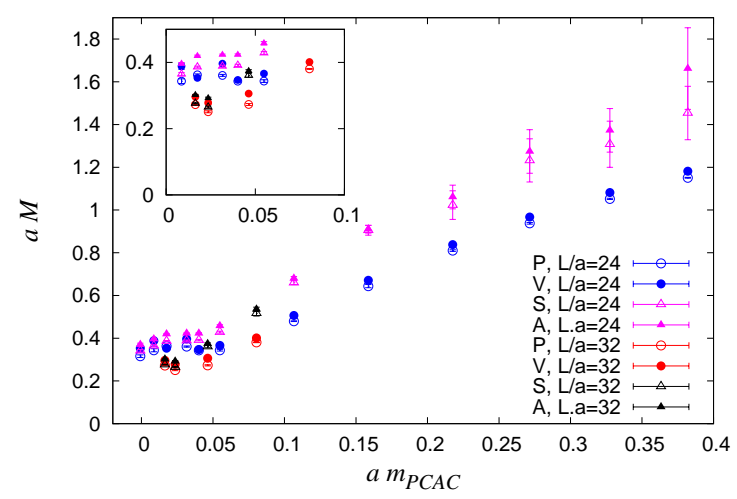

Figure 5: Masses of lightest pseudoscalar $(\mathrm{P})$, vector $(\mathrm{V})$, scalar $(\mathrm{S})$ and axial-vector $(\mathrm{A})$ mesons for $L / a=24$ and $L / a=32$.

For $L / a=8$, we also checked that the property (1) persists for Iwasaki gauge action and/or clover fermions, and that $a m_{\psi}^{\text {sat }}(L / a=8)$ depends modestly on the types of action and $\beta$. This saturation phenomenon was also observed in $\mathrm{SU}(3)_{\mathrm{C}}$ gauge theory with two sextet quarks [16]. When we look at Figure 3 from large $a m_{\mathrm{PCAC}}$, the meson masses branch at larger $\operatorname{am}_{\mathrm{PCAC}}$ with $\operatorname{am}_{\psi}^{\mathrm{sat}}(L / a)$ for smaller $L / a$, which strongly indicates that this saturation originates from finite size effect.

We comment on the dependence of $a m_{\psi}^{\text {sat }}(L / a)$ on the linear size $L / a$ of the system. The spatial correlation length $\xi_{\psi}$ cannot become larger than the system size $L / a$ and saturated at $\sim L / a$. Thus, if the Compton wavelength $2 \pi /\left(a m_{\psi}\right)$ coincides with $\xi_{\psi}, \operatorname{am}_{\psi}^{\text {sat }}(L / a)$ then decreases with $L / a$, accounting for the dependence of $a m_{\psi}^{\text {sat }}(L / a)$ on $L / a$ in Figure $3^{2}$. However, this is actually not the case in $\mathrm{SU}(2)_{\mathrm{C}}$ gauge theory with two adjoint Dirac fermions, where $a m_{\mathrm{P}}^{\text {sat }}(L / a)$ increases with $L / a$ [18]. We recall that the finite size correction consists of two terms [19]; the term induced by $P P$ scattering which increases the pseudoscalar meson mass $a m_{P}$, and the one induced by the propagation of $0^{++}$which decreases $a m_{P}$. Since the two adjoint fermion system contains a glueball-

\footnotetext{
${ }^{2}$ The finite size effect observed here differs from the power correction found in Ref. [17].
} 
rich $0^{++}$lighter than $P[20]$, the latter contribution is possibly larger than the former. The decrease of $\operatorname{am}_{\mathrm{P}}^{\mathrm{sat}}(L / a)$ with $L / a$ in our target system suggests that the $P P$ scattering contribution is the dominant source of the finite size effect. The knowledge on the mass of the lightest $0^{++}$will surely give us more comprehensive understanding on this issue.

Our interest is if the property (2) reflects the dynamics of two-color QCD with six flavors of almost massless quarks. The high degeneracy in masses between vector and pseudoscalar mesons was also observed in the $\mathrm{SU}(2)_{C}$ gauge theory with two adjoint fermions [18], which is considered to be conformal in the deep IR. Meanwhile, such a degeneracy reminds us the spectrum of bound states of massive quarks, $m_{q} \gg \Lambda_{\mathrm{QCD}}$. Another possibility is that it may occur as a consequence of finite size effect; even if the theory is confining, the system size is too small for the confining force to act between quark and anti-quarks so that they are bounded solely by $\mathbb{Z}$-copies of Coulombic forces. The knowledge on the heavy quark potential will help to answer these questions.
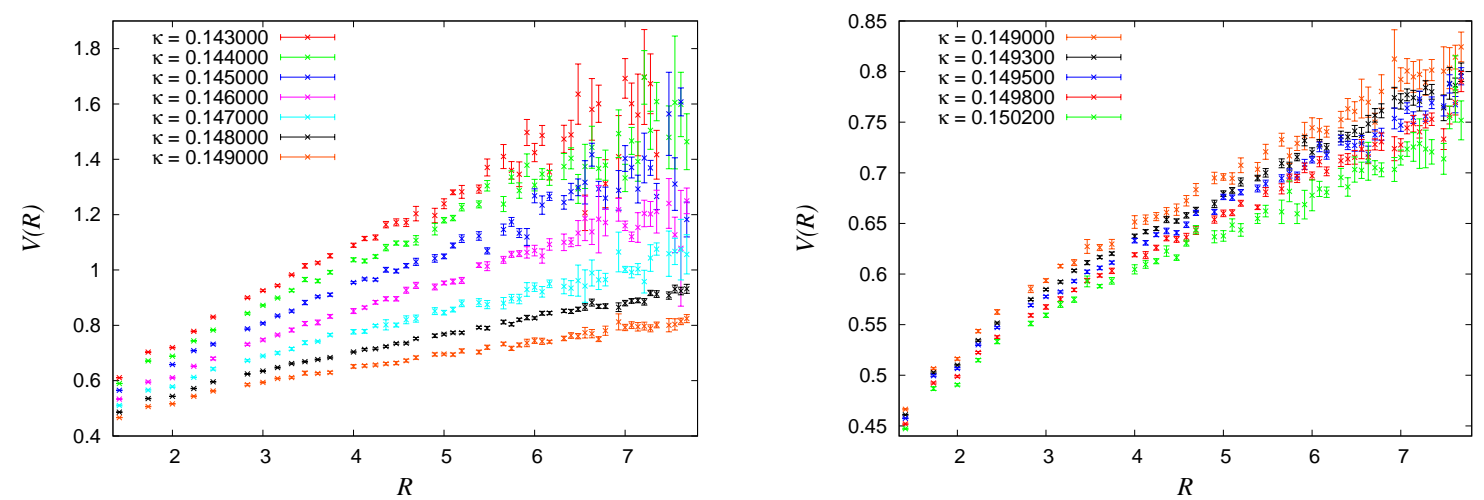

Figure 6: Heavy quark potential obtained from the lattice with $24^{3} \times 48, \beta=2.0,0.1490 \leq \kappa \leq 0.1498$ (left panel), and $0.1498 \leq \kappa \leq 0.1502$ (right panel). Note that the scale of the ordinate of the right differs from that in the left.

The heavy quark potential is extracted from the measurement of various sizes of Wilson loops. To reduce short distance fluctuation, four-level Wilson flow smearing [21] with the weight $\varepsilon=0.01$ in the exponent was performed for link variables in the spatial directions. Figure 6 plots the heavy quark potentials on the lattices with $L / a=24$ and various $\kappa$. See Figure 4 for the correspondence of $1 / \kappa$ and $a m_{\mathrm{PCAC}}$. We can see that the heavy quark potential contains the component of linear term, i.e., confinement.

Figure 7 shows the squared root $a \sqrt{\sigma}$

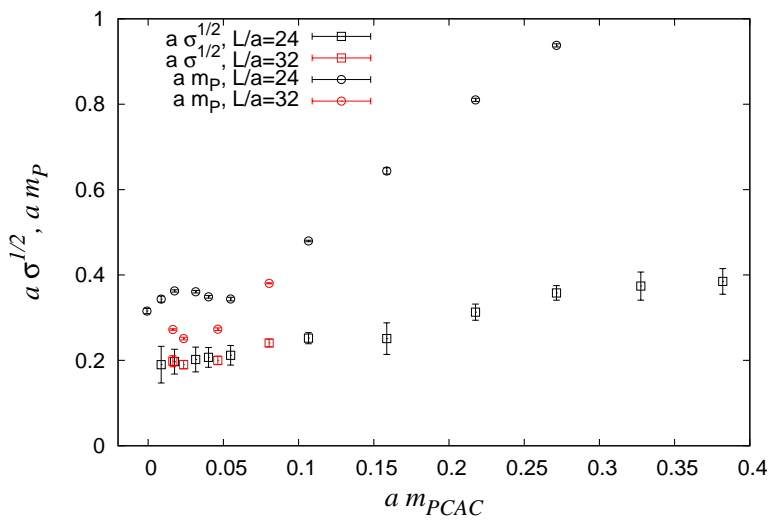

Figure 7: Masses of lightest pseudoscalar (P) and squared root of string tension $a \sqrt{\sigma}$. of the string tension. The comparison of $a \sqrt{\sigma}$ for $L / a=24$ with that for $L / a=32$ indicates that the finite size effect is not substantial for string tension. Moreover, there is no such a supporting evidence that it approaches to zero in the limit $a m_{\mathrm{PCAC}} \rightarrow 0$, suggesting that the theory is confining 
in the chiral limit. However, $a m_{\mathrm{P}}$ relative to $a \sqrt{\sigma}$ in Figure 7 cautions that if the system exhibits spontaneous chiral symmetry breakdown, the simulation on the lattice, say with size $L / a=48$, will be necessary to capture genuine chiral dynamics.

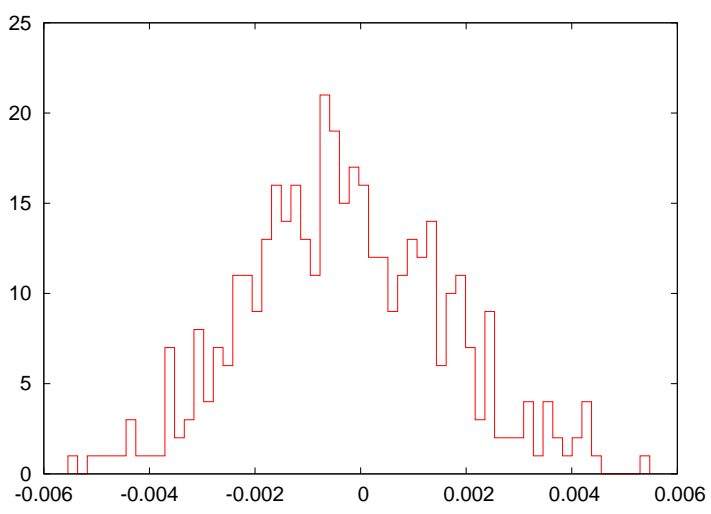

Figure 8: Distribution of Polyakov loops in the $z$-direction $\left(N_{Z}=32\right.$, periodic) on the lattice with $32^{3} \times 64, \beta=2.0, \kappa=0.14965$.

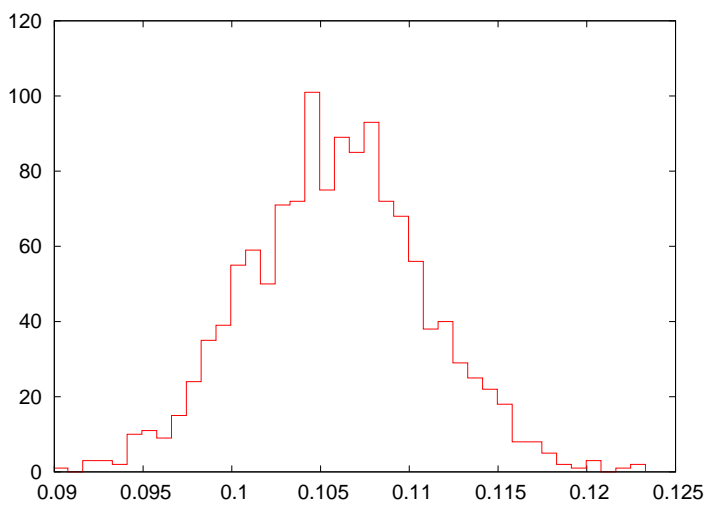

Figure 9: Distribution of Polyakov loops in the direction $\left(N_{T}=8\right.$ and anti-periodic) on the lattice with $18^{2} \times 48 \times 8, \beta=2.0, \kappa=0.1492$.

Lastly, we study the distribution of Polyakov loop $P$ in the thermal direction. $P$ takes its value in a real number in the $\mathrm{SU}(2)$ gauge theory. Its vacuum expectation value $\langle P\rangle$ is not a good measure of confinement/deconfinement transition in presence of dynamical quarks in the fundamental representation, which explicitly breaks the center $\mathbb{Z}_{2}$. In spite of this fact, its distribution will help to capture the essence of the dynamics; the system is deconfining if the distribution of $P$ does not cover 0 and is completely asymmetric with respect to the origin, while it is confining if it peaks at the origin and distributes almost symmetrically.

We can see that the periodic boundary condition is equivalent to the anti-periodic boundary condition in $\mathrm{SU}(2)_{C}$ gauge theory if all matter fields belong to the representations of $\mathbb{Z}_{2}$-odd conjugacy classes. (Only the sign of a $\mathbb{Z}_{2}$-odd observable depends on the condition.) Thus, we regard one of the spatial directions with 32 sites on lattices of $32^{3} \times 64$ as a thermal direction and measure $P$ along this direction. Figure 8 shows the distribution of $P$ for $\kappa=0.14965$. The periodic/antiperiodic boundary condition will reduce the dynamical modes so that the finite size effect will act to order the system $(\langle P\rangle \neq 0$, i.e., tendency of deconfinement). Even though the aspect ratio is 32/32, the distribution of $P$ in Figure 8 exhibits disordering, suggesting confinement. In contrast, the distribution of $P$ for $N_{T}=8$ in Figure 9 does not cover 0 , suggesting deconfinement. The question whether this transition is really thermal type needs further study.

To summarize, we show some evidence supporting confinement in the two-color QCD with six-flavors of quarks. As can be seen in the inset of Figure 3, the mass splitting between pseudoscalar meson and vector meson increases gradually in the small quark mass region until saturation is encountered. The study with larger size of lattices is necessary to approach smaller $m_{\mathrm{P}} / \sqrt{\sigma}$ and to get a definite conclusion. Now, it is interesting to start the calculation of the other physical quantities, such as the mass of the lightest particle in the $0^{++}$channel relative to the decay constant. 


\section{Acknowledgments}

We thank A. Patella and L. Del Debbio for discussion on their work in [18]. This work is supported in part by Grants-in-Aid for Scientific Research (S)22224003, (C)20540261, (A)22244018, (C)24540276, Grant-in-Aid for Young Scientists (B)23740177, (B)22740183, and Grant-in-Aid for Scientific Research on Innovative Areas 20105001, 20105002, 20105005, 23105707. The computation on the large lattices $(L / a=16,18,24)$ was conducted on the supercomputer system $\varphi$ at Nagoya University, and on the servers with GPGPUs at KEK.

\section{References}

[1] T. Appelquist, G. T. Fleming and E. T. Neil, Phys. Rev. Lett. 100 (2008) 171607 [Erratum-ibid. 102 (2009) 149902].

[2] T. Appelquist, et al., arXiv:1204.6000 [hep-ph].

[3] M. Hayakawa, K. -I. Ishikawa, Y. Osaki, S. Takeda, and N. Yamada, in preparation.

[4] Z. Fodor, K. Holland, J. Kuti, D. Nogradi and C. Schroeder, Phys. Lett. B 681 (2009) 353.

[5] A. Hasenfratz, Phys. Rev. Lett. 108 (2012) 061601.

[6] M. E. Peskin, Nucl. Phys. B 175 (1980) 197; J. Preskill, Nucl. Phys. B 177 (1981) 21.

[7] B. Svetitsky and L. G. Yaffe, Nucl. Phys. B 210 (1982) 423.

[8] Y. Iwasaki, K. Kanaya, S. Kaya, S. Sakai and T. Yoshie, Phys. Rev. D 69 (2004) 014507.

[9] F. Bursa, L. Del Debbio, L. Keegan, C. Pica and T. Pickup, Phys. Lett. B 696 (2011) 374.

[10] G. Voronov [LSD Collaboration], PoS LATTICE 2011 (2011) 093.

[11] T. Karavirta, J. Rantaharju, K. Rummukainen and K. Tuominen, JHEP 1205 (2012) 003.

[12] H. Ohki, et al., PoS LATTICE 2010 (2010) 066.

[13] M. Luscher, R. Narayanan, P. Weisz and U. Wolff, Nucl. Phys. B 384 (1992) 168 S. Sint, Nucl. Phys. B 421 (1994) 135

[14] Y. Shamir, B. Svetitsky and T. DeGrand, Phys. Rev. D 78 (2008) 031502.

[15] M. Hayakawa, K. -I. Ishikawa, Y. Osaki, S. Takeda, S. Uno and N. Yamada, Phys. Rev. D 83 (2011) 074509.

[16] T. DeGrand, Y. Shamir and B. Svetitsky, Phys. Rev. D 79 (2009) 034501.

[17] M. Fukugita, H. Mino, M. Okawa, G. Parisi and A. Ukawa, Phys. Lett. B 294 (1992) 380.

[18] L. Del Debbio, B. Lucini, A. Patella, C. Pica and A. Rago, PoS LATTICE 2011 (2011) 084.

[19] M. Lüscher, Commun. Math. Phys. 104 (1986) 177.

[20] L. Del Debbio, B. Lucini, A. Patella, C. Pica and A. Rago, Phys. Rev. D 82 (2010) 014510.

[21] M. Lüscher, Commun. Math. Phys. 293 (2010) 899. 